Modelling, Analysis and Simulation

MAS Modelling, Analysis and Simulation

Casimir scaling, glueballs, and hybrid gluelumps

V. Mathieu, C. Semay, F. Brau

RePORT MAS-R0604 February 2006 
Centrum voor Wiskunde en Informatica (CWI) is the national research institute for Mathematics and Computer Science. It is sponsored by the Netherlands Organisation for Scientific Research (NWO).

CWI is a founding member of ERCIM, the European Research Consortium for Informatics and Mathematics.

CWI's research has a theme-oriented structure and is grouped into four clusters. Listed below are the names of the clusters and in parentheses their acronyms.

Probability, Networks and Algorithms (PNA)

Software Engineering (SEN)

\section{Modelling, Analysis and Simulation (MAS)}

Information Systems (INS)

Copyright (C) 2006, Stichting Centrum voor Wiskunde en Informatica

P.O. Box 94079, 1090 GB Amsterdam (NL)

Kruislaan 413, 1098 SJ Amsterdam (NL)

Telephone +31205929333

Telefax +31205924199

ISSN 1386-3703 


\title{
Casimir scaling, glueballs, and hybrid gluelumps
}

\begin{abstract}
Assuming that the Casimir scaling hypothesis is well verified in QCD, masses of glueballs and hybrid gluelumps (gluon attached to a point-like $c \bar{c}$ pair) are computed within the framework of the rotating string formalism. In our model, two gluons are attached by an adjoint string in a glueball, while the gluon and the colour octet $c \bar{c}$ pair are attached by two fundamental strings in a hybrid gluelump. Masses for such exotic hadrons are computed with very few free parameters. These predictions can serve as a guide for experimental searches. In particular, the ground state glueballs lie on a Regge trajectory and the lightest $2^{++}$state has a mass compatible with some experimental candidates.
\end{abstract}

2000 Mathematics Subject Classification: 81V05; 81V35

Keywords and Phrases: Glueball; Relativistic quark model

Note: This work was carried out under project MAS3 | NWO 633.000.401 



\title{
Casimir scaling, glueballs, and hybrid gluelumps
}

\author{
Vincent Mathieu $^{\mathrm{a} 1}$, Claude Semay ${ }^{\mathrm{b} 1}$ and Fabian Brau ${ }^{2}$ \\ 1 Groupe de Physique Nucléaire Théorique, Université de Mons-Hainaut, Académie universitaire Wallonie-Bruxelles, Place du \\ Parc 20, BE-7000 Mons, Belgium, e-mail: vincent.mathieu@umh.ac.be, claude.semay@umh.ac.be \\ 2 CWI, P.O. Box 94079, 1090 GB Amsterdam, The Netherlands, e-mail: f.brau@cwi.nl
}

January 31, 2006

\begin{abstract}
Assuming that the Casimir scaling hypothesis is well verified in QCD, masses of glueballs and hybrid gluelumps (gluon attached to a point-like $c \bar{c}$ pair) are computed within the framework of the rotating string formalism. In our model, two gluons are attached by an adjoint string in a glueball, while the gluon and the colour octet $c \bar{c}$ pair are attached by two fundamental strings in a hybrid gluelump. Masses for such exotic hadrons are computed with very few free parameters. These predictions can serve as a guide for experimental searches. In particular, the ground state glueballs lie on a Regge trajectory and the lightest $2^{++}$state has a mass compatible with some experimental candidates.
\end{abstract}

PACS. 12.39.Mk Glueball and nonstandard multiquark gluon states - 12.39.Ki Relativistic quark model - 12.39.Pn Potential model

\section{Introduction}

Lattice calculations $[1,2]$ and other models of QCD $[3,4]$ predict that the Casimir scaling hypothesis is well verified in QCD, that is to say that the potential between two opposite colour charges in a colour singlet is proportional to the value of the quadratic Casimir operator. This mechanism can be tested for instance in two-gluon glueballs. Direct evidence for such a system is still controversial, but several models [5-8] predict similar masses with values close to some of the experimental candidates $[9,10]$. The Casimir scaling hypothesis can also be tested in another system whose colour-spin structure is similar to the one of a glueball. Let us consider an hybrid meson containing a colour octet spin one $c \bar{c}$ pair and a gluon. Due their very heavy masses, the charm quarks can be assumed nearly fixed at the centre of mass with the gluon orbiting around. A gluon attached to a static colour octet ("infinitely heavy gluon" approximation) is called a gluelump $[11,12]$, so the $g c \bar{c}$ system considered here is called a hybrid gluelump as in Ref. [13].

In our model, a quark and an antiquark are attached by a fundamental string while two gluons in a glueball are attached by an adjoint string. We assume here that the three-body nature of the confinement implies that the gluon and the colour octet pair are attached by two fundamental strings in a hybrid gluelump. Within these hypothesis, we compute the masses of glueballs and hybrid gluelumps with a simple effective model of QCD [1416]. This semirelativistic potential model is described in

${ }^{a}$ IISN Scientific Research Worker

b FNRS Research Associate
Sec. 2. It depends on few parameters which are fixed on well known mesons in Sec. 3. Masses of glueballs and hybrid gluelumps are computed respectively in Sec. 4 and 5. We focus our attention on the first orbital excitations, neglecting the details of the spin dependent interactions, assumed weak with respect to the confinement. Some concluding remarks are given in Sec. 6 .

\section{Hamiltonian}

Starting from the QCD theory, a Lagrangian for a system of two confined spinless colour sources can be derived taking into account the dynamical degrees of freedom of the string, with tension $\sigma$, joining the two particles: the rotating string model (RSM) [14-16]. This model is completely equivalent to the relativistic flux tube model [17], once the auxiliary fields appearing in the RSM are properly eliminated [18-20]. The nonlinear coupled equations of these models are difficult to solve $[17,21]$. So we will use an approximation of these effective QCD theories [22,23], which is simply given by the following spinless Salpeter Hamiltonian

$$
H_{0}=\sqrt{\boldsymbol{p}^{2}+m_{1}^{2}}+\sqrt{\boldsymbol{p}^{2}+m_{2}^{2}}+\sigma r,
$$

completed by a perturbative part due to the motion of the string

$$
\begin{aligned}
& \Delta H_{\mathrm{str}}=-\frac{\sigma L(L+1)}{r} \\
& \times \frac{\left[4\left(\mu_{1}^{2}+\mu_{2}^{2}-\mu_{1} \mu_{2}\right)+\left(\mu_{1}+\mu_{2}\right) \sigma r\right]}{2 \mu_{1} \mu_{2}\left[12 \mu_{1} \mu_{2}+4\left(\mu_{1}+\mu_{2}\right) \sigma r+(\sigma r)^{2}\right]} .
\end{aligned}
$$


The quantity $\mu_{i}$ appearing in the above equation is a kind of constituent particle mass given by

$$
\mu_{i}=\left\langle\sqrt{\boldsymbol{p}^{2}+m_{i}^{2}}\right\rangle
$$

in which the average value is taken for an eigenstate of the Hamiltonian (1). The constituent mass is then statedependent. Equation (2) is obtained by taking the slow motion limit of Eqs. (33) in Ref. [20]. The contribution $\Delta M_{\text {str }}$ to the mass due to the string can be computed with a good precision by the following approximation $[22$, 23]

$$
\begin{aligned}
& \Delta M_{\text {str }}=-\sigma L(L+1)\left\langle\frac{1}{r}\right\rangle \\
& \times \frac{\left[4\left(\mu_{1}^{2}+\mu_{2}^{2}-\mu_{1} \mu_{2}\right)+\left(\mu_{1}+\mu_{2}\right) \sigma\langle r\rangle\right]}{2 \mu_{1} \mu_{2}\left[12 \mu_{1} \mu_{2}+4\left(\mu_{1}+\mu_{2}\right) \sigma\langle r\rangle+(\sigma\langle r\rangle)^{2}\right]} .
\end{aligned}
$$

It is worth noting that, in this formalism, the particle masses $m_{1}$ and $m_{2}$ are the current ones. So, in the following, we will consider that the mass $m_{n}$ of the $n$ quark ( $n$ stands for $u$ or $d$ ) and the mass $m_{g}$ of the gluon are vanishing.

A significant contribution to hadron masses is also given by the one-gluon exchange (OGE) mechanism between colour sources. At the zero order (neglecting the spin), the interaction has the following form

$$
\Delta H_{\text {Coul }}=-\kappa \frac{\alpha_{S}}{r} .
$$

$\alpha_{S}$ is a phenomenological strong coupling constant and $\kappa$ is a colour factor given by

$$
\kappa=\frac{1}{2}\left(C_{12}-C_{1}-C_{2}\right),
$$

where $C_{12}$ is the colour Casimir operator for the pair 12 and $C_{i}$ is the corresponding one for the particle $i$.

If $M_{0}$ is the solution of the eigenequation $H_{0}|\phi\rangle=$ $M_{0}|\phi\rangle$, the total mass $M$ for the system of the two colour sources is given by

$$
M=M_{0}+\Delta M_{\text {str }}+\left\langle\Delta H_{\text {Coul }}\right\rangle .
$$

In this formula, the Coulomb-like contribution is computed as a perturbation. It is shown in Ref. [22], that this approximation is very good, especially for states with high angular momentum $L$. All computation are performed using the Lagrange-mesh method [24].

Recently, it was shown that the quark self-energy (QSE) contribution, which is created by the colour magnetic moment of the quark propagating through the vacuum background field, adds a negative constant to the hadron masses [25]. Its negative sign is due to the paramagnetic nature of the particular mechanism at work in this case. Other contributions due to quark spin (spin-spin, spin-orbit) also exist but they are smaller that the QSE one [14], and they are neglected in this work. The QSE contribution $\Delta S_{i}$ for a quark of current mass $m_{i}$ is given by

$$
\Delta S_{i}=-f \frac{\sigma}{2 \pi} \frac{\eta\left(m_{i} / \delta\right)}{\mu_{i}} .
$$

The $\eta$ function is such that $\eta(0)=1$, and its value decreases monotonically towards 0 with increasing quark mass [23]. $\delta$ is the inverse of the gluonic correlation length, and its value is estimated about $1.0-1.3 \mathrm{GeV}$. As the meson masses vary very little with this parameter [23], we fix the value of $\delta$ at $1 \mathrm{GeV}$ (meson masses do not depend on $\delta$ when $m=0$ ). The factor $f$ has been computed by lattice calculations. First quenched calculations gave $f=4$ [26]. A more recent unquenched work [27] gives $f=3$, the value that we choose in this work. If a hadron contains $q$ quarks, a contribution $\sum_{i=1}^{q} \Delta S_{i}$ must be added to its mass. Strong theoretical and phenomenological arguments indicate that gluons do not bring any contribution of self energy $[28,29]$.

\section{Parameters}

As we are mainly interested in describing the main features of spectra, we can use a Hamiltonian of spinless particles since spin effects are generally an order of magnitude smaller than orbital or radial excitations. Moreover, we will consider hadrons for which the spin-dependent part of the Hamiltonian is the weakest possible.

The raise of degeneracy between spin 0 and spin 1 mesons can be due to a spin-spin potential coming from the OGE or due to an instanton induced interaction [30, 31]. For spin 1 mesons, the spin-dependent interaction is small in the former case or vanishing in the latter case. The spin-dependent part of the gluon-gluon interaction has a small contribution for glueballs with spin 2 [8]. If we consider a hybrid gluelump containing a $c \bar{c}$ pair with spin 1, we minimize the spin effect for the heavy quarks. As such a system is similar to a glueball from the point of view of the spin-colour structure, we can also expect that the spin contribution will be the smallest if we study spin 2 hybrids. For all these reasons, as in Ref. [13], we will only consider systems with maximal value of the total spin $S: J=L+1$ for mesons and $J=L+2$ for glueballs and hybrid gluelumps.

A correct determination of the parameter is crucial to predict new states. For theoretical reasons, we have chosen to take $m_{n}=0, m_{g}=0, f=3$, and $\delta=1 \mathrm{GeV}$. Now, we will fix the values of the remaining free parameters with well established meson states. As, the light $n \bar{n}$ spin 1 mesons can be isoscalar $(I=0)$ or isovector $(I=1)$, we will use an average mass defined, as usual, by

$$
M_{n \bar{n}}(\mathrm{av} .)=\frac{M_{n \bar{n}}(I=0)+3 M_{n \bar{n}}(I=1)}{4} .
$$

At this stage, it is interesting to obtain an approximate analytical formula for light $n \bar{n}$ mesons. For such a system, $m_{1}=m_{2}=0$ and $\mu_{1}=\mu_{2}=\mu=\left\langle\sqrt{\boldsymbol{p}^{2}}\right\rangle$. It can then be shown that $M_{0}=4 \mu$ with $\mu \propto \sqrt{\sigma}$ [19]. Using the semirelativistic virial theorem [32], we find $\langle r\rangle=2 \mu / \sigma$. With the following approximation $\langle 1 / r\rangle \approx 1 /\langle r\rangle$, we obtain finally

$$
M_{n \bar{n}} \approx \sqrt{\sigma}\left(4 \nu-\frac{L(L+1)}{16 \nu^{3}}-\frac{\kappa \alpha_{S}}{2 \nu}-\frac{f}{\pi \nu}\right)
$$


Table 1. Experimental masses in $\mathrm{GeV}$ for some light mesons $(n \bar{n}), D^{*}$ mesons $(c \bar{n})$, and charmoniums $(c \bar{c})$, with maximal $S$ and $J=L+S$ [33]. The mesons $\psi(4040)$ and $\psi(4415)$ are assigned to $N=2$ and $N=3$ states respectively.

\begin{tabular}{lrrrr}
\hline$N=0, J^{P(C)}$ & $1^{-(-)}$ & $2^{+(+)}$ & $3^{-(-)}$ & $4^{+(+)}$ \\
\hline$n \bar{n} I=0$ & 0.783 & 1.275 & 1.667 & 2.034 \\
$n \bar{n} I=1$ & 0.775 & 1.318 & 1.689 & 2.001 \\
$n \bar{n}$ av. & 0.777 & 1.307 & 1.684 & 2.009 \\
$c \bar{n}$ & 2.010 & 2.460 & & \\
$c \bar{c}$ & 3.097 & 3.556 & & \\
\hline$J=1, N$ & 0 & 1 & 2 & 3 \\
\hline$c \bar{c}$ & 3.097 & 3.686 & 4.040 & 4.415 \\
\hline
\end{tabular}

in which $\nu=\mu / \sqrt{\sigma}$ is independent of $\sigma$. Let us note that an approximate value for $\nu$ is given by [23]

$$
\nu \approx\left(\frac{\epsilon_{N L}}{3}\right)^{3 / 4}
$$

where $\epsilon_{N L}$ is the solution of the dimensionless Hamiltonian $\boldsymbol{q}^{2}+|\boldsymbol{x}|$ in which $\boldsymbol{q}$ and $\boldsymbol{x}$ are conjugate variables. The accuracy of formula (10) with the approximation (11) is about $5-10 \%[22]$.

The contribution of the motion of the string to the meson mass is small, since the ratio $\Delta M_{\text {str }} / M_{0}$ is at most $1 / 16$ [23]. Formula (10) shows that the contribution of the Coulomb term and of the self energy shift the square mass $M_{n \bar{n}}^{2}$ of the meson without modifying the slope of the Regge trajectories [23]. This slope depends mainly on the string tension which we call $a$ for a quark-antiquark bound state. With a fit, it is only possible to determine the following combination of parameters $\kappa \alpha_{S} / 2-f / \pi$. But since the value of $f$ is fixed, it is possible to compute $\alpha_{S}$.

We fix the parameters by fitting the results, obtained with formula (7) and the exact solutions of Hamiltonian (1), on experimental data [33] (see Table 1). Using the Regge trajectory for light meson, we find $a=0.175 \mathrm{GeV}^{2}$ and $\alpha_{s}=0.10$. The value obtained for $\alpha_{S}$ is small with respect to values found in other potential models of meson. For instance, in Ref. [22], a value of 0.39 is obtained because the meson masses are fitted on the centre of gravity of spin 1 and spin 0 mesons. In this case, the average mass of the $J=1 n \bar{n}$ state is $0.612 \mathrm{GeV}$, a value quite different from the one we choose, $0.777 \mathrm{GeV}$. The mass of the quark $c$ is determined by computing the masses of $D^{*}$ mesons, which are systems dynamically closer to hybrid gluelumps than charmonium states. All parameters are gathered in Table 2 .

The quality of a fit is estimated by computing for each state the relative error on binding energy. The theoretical (experimental) binding energy for a meson is given by the theoretical (experimental) mass minus the theoretical current masses of the quark and the antiquark ( 0 for the light $n \bar{n}$ mesons, $m_{c}$ for the $D^{*}, 2 m_{c}$ for the charmoniums). Quite good meson masses are obtained, taking into account the simplicity of the model (see Table 3 ). The error is around $1 \%$ for light $n \bar{n}$ mesons (see Fig. 1), around
Table 2. Parameters of the model. The values of $m_{n}, m_{g}$, $m_{c \bar{c}}, f$, and $\delta$ are fixed by theoretical or phenomenological considerations (see text). The string tension $a$ for a meson and the strong coupling constant $\alpha_{S}$ are free parameters.

\begin{tabular}{ll}
\hline$m_{n}=0$ & $a=0.175 \mathrm{GeV}^{2}$ \\
$m_{g}=0$ & $\alpha_{s}=0.10$ \\
$m_{c}=1.300 \mathrm{GeV}$ & $f=3$ \\
$m_{c \bar{c}}=2 m_{c}$ & $\delta=1 \mathrm{GeV}$ \\
\hline
\end{tabular}

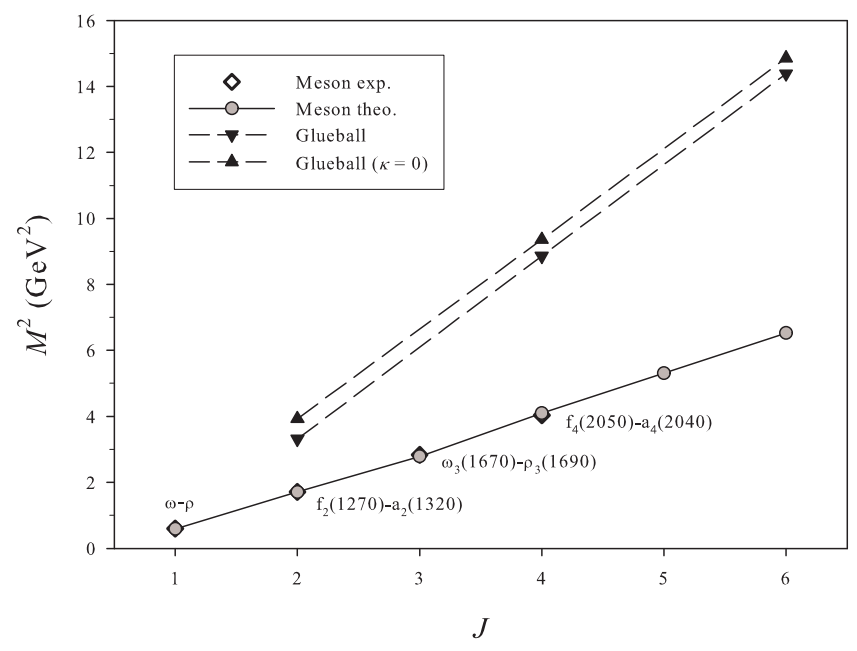

Fig. 1. Regge trajectories for light mesons (average between $I=0$ and $I=1$ ) and glueballs (with or without Coulomb interaction). These states are characterized by $N=0, S$ maximal, and $J=L+S$.

$3 \%$ for the $D^{*}$ (see Fig. 2), around $10 \%$ for the mesons $J / \psi$ and $\chi_{c 2}$ (see Fig. 2), but less than $5 \%$ for radial excitations of the $J / \psi$ (see Fig. 3).

\section{Glueballs}

In this paper, we assume that the string tension between two colour sources is controlled by the Casimir scaling. So, contrary to what is done in Ref. [13], we assume that the ratio between the string tension in a glueball and the string tension in a meson is given by the ratio of the Casimir operators. In a two gluon system, we have then $\sigma=9 a / 4$, and the Regge slope is increased by the same factor. Moreover, following the theory of Simonov [28, 29], there is no self-energy contribution coming from gluon. Let us note that, in the same framework [14-16], the constituent mass of a particle is directly proportional to $\sqrt{\sigma}$ when its current mass $m$ is vanishing. If we compute $\mu_{g}$ from a two-gluon glueball and $\mu_{n}$ from a light meson, we have $\mu_{g} / \mu_{n}=3 / 2$. A gluon cannot decay spontaneously into a $n \bar{n}$ pair.

At the lowest order, the colour Coulomb factor in such a system is given by the formula (6), that is to say $\kappa=3$. Nevertheless, in some model, it is found that the Coulomb 


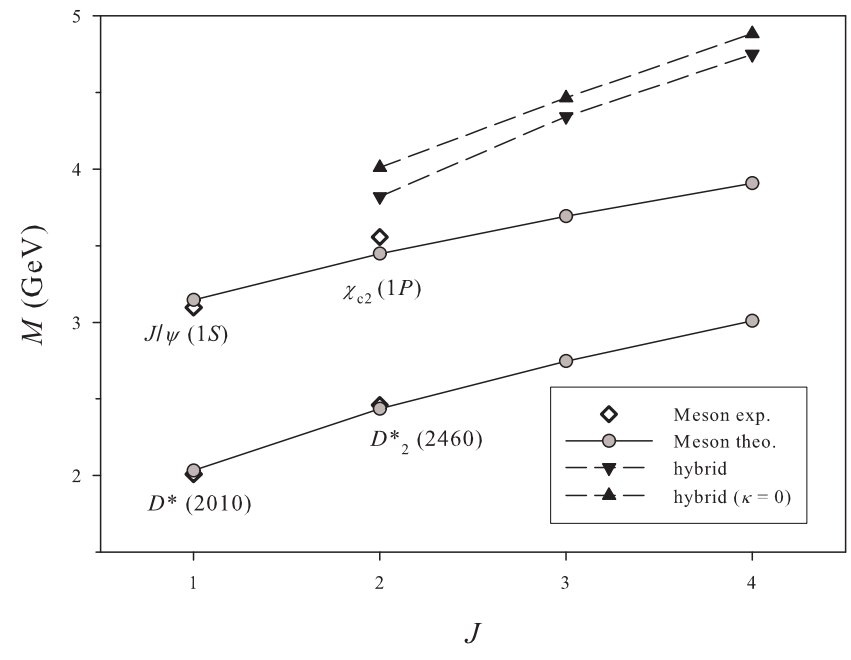

Fig. 2. Masses for $c \bar{n}, c \bar{c}$, and hybrid gluelumps (with or without Coulomb interaction). These states are characterized by $N=0, S$ maximal, and $J=L+S$.

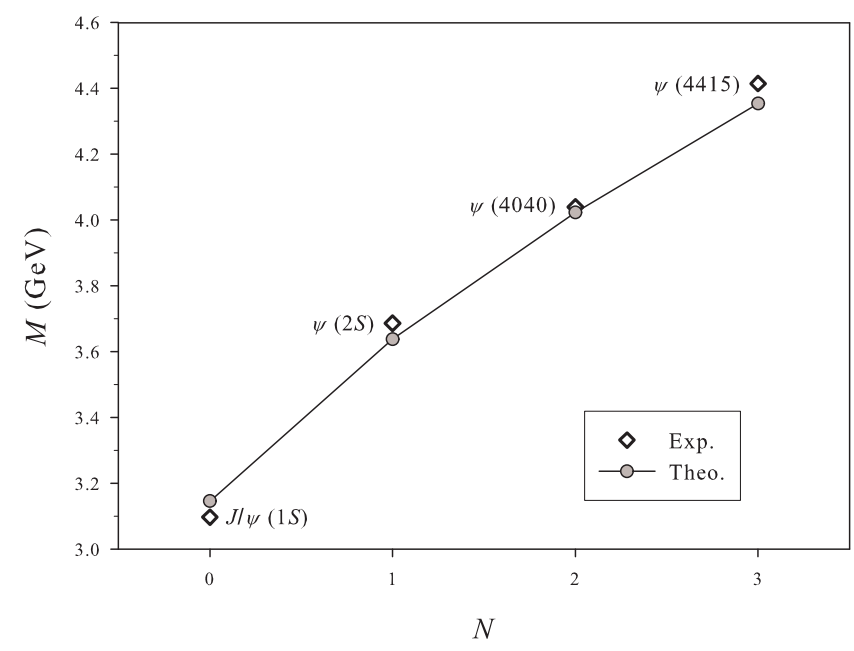

Fig. 3. Masses of $c \bar{c} J=1$ mesons as a function of the quantum radial number $N$. The states $\psi(4040)$ and $\psi(4415)$ are assigned to $\psi(3 S)$ and $\psi(4 S)$ respectively.

force in glueballs could be damped [5] or strongly reduced [28]. So, we have considered both cases $\kappa=0$ and $\kappa=3$ to study this possible effect. As our value of $\alpha_{S}$ is small, the two spectra do not differ strongly.

The colour wave function of two gluons is symmetric and the spin wave function considered here is symmetric since $S=2$. As gluons are bosons, the spatial wave function is also symmetric and $L$ is even. The first three states are computed with the six lowest light mesons of the first Regge trajectory. Results, obtained with formula (7) and the exact solutions of Hamiltonian (1), are given in Table 3 and in Fig. 1. When $\kappa=3$, the trajectory is

$$
J=0.36 M^{2}+0.80 \text {. }
$$

Table 3. Predicted masses in $\mathrm{GeV}$ for $n \bar{n}$ (average between $I=0$ and $I=1$ ), $c \bar{n}, c \bar{c}$, glueballs (only $L$ even), and hybrid gluelumps. These states are characterized by $N=0, S$ maximal, and $J=L+S$. The asterisk indicates that the states is calculated with $\kappa=0$. Only the three first states are indicated for glueballs and hybrids.

\begin{tabular}{lrrrrrr}
\hline$J$ & 1 & 2 & 3 & 4 & 5 & 6 \\
\hline$n \bar{n}$ & 0.767 & 1.306 & 1.670 & 2.024 & 2.304 & 2.555 \\
Glueball & - & 1.820 & - & 2.976 & - & 3.793 \\
Glueball* & - & 1.981 & - & 3.059 & - & 3.855 \\
$c \bar{n}$ & 2.033 & 2.436 & 2.747 & 3.010 & & \\
$c \bar{c}$ & 3.146 & 3.449 & 3.693 & 3.908 & & \\
Hybrid & - & 3.820 & 4.343 & 4.749 & & \\
Hybrid* & - & 4.010 & 4.464 & 4.884 & & \\
\hline
\end{tabular}

When $\kappa=0$, the slope is not changed and the intercept becomes 0.57 . This results is somewhat different from the results of Refs. [13,34] because the smaller value of our string tension $a$. But our glueball masses are compatible with those of the model III.A of Ref. [8]. Our predictions are also in good agreement with the spin averaged glueball masses of Ref. [28]. The model in this last paper and our model rely on the same physical bases. More states are predicted in Ref. [28] because more values are considered for the spin and the radial quantum number.

Moreover, our lightest state has a mass compatible with possible experimental candidates $[9,10]$.

\section{Hybrids}

With formula (6), we find $\kappa=3 / 2$ for a gluon-quark pair inside the hybrid. As the quark and the antiquark are assumed to be very close, the total colour factor for the Coulomb-like force between the gluon and the point-like $c \bar{c}$ pair is $2 \times 3 / 2=3$, which is the same as into a two gluon glueball. As in the previous case, we have considered both cases $\kappa=0$ and $\kappa=3$.

The situation is different for the confinement which is actually a three-body force. Let us consider the general case of a triangle formed by three colour sources named $A$, $B, C$, and a point $P$ inside this triangle, where the three flux tubes, with lengths $|A P|,|B P|,|C P|$, generated by these sources meet [35]. The triangular inequalities imply $|A B|+|A C|<2|A P|+|B P|+|C P|$. Let us assume that the energy of these flux tubes are respectively $\sigma_{A}|A P|, \sigma|B P|$, and $\sigma|C P|$, with $\sigma_{A}=k \sigma$. The total energy of the flux tubes is then $\sigma(k|A P|+|B P|+|C P|)$. If $k>2$, the above inequality shows that it is energetically favourable for the junction point $P$ to be on the source $A$. The potential energy of the system is then $\sigma(|A B|+|A C|)$. In the case of a hybrid gluelump, the gluon is at position $A$ with $k=$ $9 / 4$ and the quarks occupy positions $B$ and $C$, which are merged here. So the confining energy is equal to two times the confining energy in a meson, and we take $\sigma=2$.

A hydrid is then considered here as a gluon and a point-like $c \bar{c}$ pair attached by two fundamental strings. To compute its mass, we must determine the mass of the 
$c \bar{c}$ pair. In our model, there is no direct confining interaction between the quark and the antiquark, but a small repulsive Coulomb force exists since formula (6) implies that $\kappa=-1 / 6$ for a quark-antiquark pair in a colour octet. The $c$ and $\bar{c}$ quarks does obviously not really occupy the same position. If we assume a typical separation around the $J / \psi$ radius, the contribution of the Coulomb interaction is around 10-20 MeV. For heavy quark, the constituent mass $\mu$ is around the current mass $m$. For a $c \bar{c}$, the contribution of the self-energy can be estimated around $-40 \mathrm{MeV}$. So, if we neglect the kinetic energy of the quarks inside the point-like $c \bar{c}$ pair, we can take the point-like $c \bar{c}$ with a mass equal to $2 m_{c}$. We estimate that this approximation can generate an error on hybrid glueball around $100 \mathrm{MeV}$.

The masses of hybrids, obtained with formula (7) and the exact solutions of Hamiltonian (1), are given up to $J=4$ with the masses of $c \bar{n}$ and $c \bar{c}$ mesons (see Table 3 and Fig. 2). The hybrid masses found are significantly lower than those obtained in Refs. [13,36], again because the smaller value of our string tension $a$. If the Coulomb interaction is cancelled, the masses increase by about $150 \mathrm{MeV}$.

A gluelump is a hypothetical object in which a real gluon is attached to a static one ("infinitely heavy gluon" approximation). The hybrid system considered here is very similar to a gluelump but with the adjoint string replaced by two fundamental strings and the infinitely heavy gluon replaced by a very heavy point-like $c \bar{c}$ pair with gluon quantum numbers. If we subtract from the hybrid masses, obtained in cancelling the Coulomb-like contribution, the quantity $2 m_{c}$, we obtain respectively $1.410,1.864$, and $2.284 \mathrm{GeV}$. These masses are in good agreement with those obtained for gluelumps within the framework of the QCD string model [11]. They are also compatible with the spectra predicted by lattice calculations $[11,37]$.

\section{Concluding remarks}

We compute the masses of the lightest glueballs and hybrid gluelumps (gluon attached to a point-like $c \bar{c}$ pair) within the framework of a simple effective model of QCD derived from the rotating string model $[14-16]$. The corresponding semirelativistic Hamiltonian is dominated by a linear confinement, supplemented by a one gluon exchange interaction and a contribution from self-energy for the quarks only. It is assumed that the Casimir scaling hypothesis is well verified, and that two gluons are attached by an adjoint string in a glueball, while the gluon and the colour octet $c \bar{c}$ pair are attached by two fundamental strings in a hybrid gluelump. Values of effective string tension $\sigma$ and colour factor $\kappa$ for the different hadrons considered here are gathered in Table 4.

In order to minimize the contributions of the spin dependent interactions, only the masses of states with maximal spin have been calculated. This strongly constraints the values of our three free parameters: the mass of the charm quark, the strong coupling constant and the string
Table 4. Values of effective string tension $\sigma$ and colour factor $\kappa$ for different hadrons.

\begin{tabular}{lrr}
\hline Hadron & $\sigma$ & $\kappa$ \\
\hline Meson & $a$ & $4 / 3$ \\
Glueball & $9 a / 4$ & 3 \\
Hybrid & $2 a$ & 3 \\
\hline
\end{tabular}

tension in a meson. With these parameters, we find glueballs and hybrid gluelumps with masses relatively small $[13,34,36]$. The contribution of the Coulomb interaction is about 100-200 MeV, depending on the particular hadron. The ground state glueballs lie on a Regge trajectory and the lightest $2^{++}$state has a mass compatible with some experimental candidates $[9,10]$. Despite the simplicity of the model, these predictions can serve as a guide for experimental searches of exotic hadrons.

\section{References}

1. S. Deldar, Phys. Rev. D 62, 034509 (2000) [heplat/9911008].

2. G. S. Bali, Phys. Rev. D 62, 114503 (2000) [heplat/0006022].

3. T. H. Hanson, Phys. Lett. B 166, 343 (1986).

4. C. Semay, Eur. Phys. J. A 22, 353 (2004) [hep-ph/0409105].

5. J. M. Cornwall, A. Soni, Phys. Lett. B 120, 431 (1983).

6. A. Szczepaniak, E. S. Swanson, C.-R. Ji, S. R. Cotanch, Phys. Rev. Lett. 76, 2011 (1996) [hep-ph/9511422].

7. C. J. Morningstar, M. Peardon, Phys. Rev. D 60, 034509 (1999).

8. F. Brau, C. Semay, Phys. Rev. D 70, 014017 (2004) [hep$\mathrm{ph} / 0412173]$.

9. B. S. Zou, Nucl. Phys. A 655, c41 (1999).

10. D. V. Bugg, M. Peardon, B. S. Zou, Phys. Lett. B 486, 49 (2000).

11. Yu. A. Simonov, Nucl. Phys. B 592, 350 (2001) [hepph/0003114].

12. N. Brambilla, A. Pineda, J. Soto, A. Vairo, Rev. Mod. Phys. 77, 1423 (2005) [hep-ph/0410047].

13. E. Abreu, P. Bicudo, hep-ph/0508281.

14. A. Yu. Dubin, A. B. Kaidalov, Yu. A. Simonov, Phys. At. Nucl. 56, 1745 (1993); [Yad. Fiz. 56, 213 (1993)] [hep$\mathrm{ph} / 9311344]$.

15. E. L. Gubankova, A. Yu. Dubin, Phys. Lett. B 334, 180 (1994) [hep-ph/9408278].

16. V. L. Morgunov, A. V. Nefediev, Yu. A. Simonov, Phys. Lett. B 459, 653 (1999) [hep-ph/9906318].

17. D. LaCourse, M. G. Olsson, Phys. Rev. D 39, 2751 (1989).

18. T. J. Allen, M. G. Olsson, Phys. Rev. D 68, 054022 (2003) [hep-ph/0306128].

19. C. Semay, B. Silvestre-Brac, I. M. Narodetskii, Phys. Rev. D 69, 014003 (2004) [hep-ph/0309256].

20. F. Buisseret, C. Semay, Phys. Rev. D 70, 077501 (2004) [hep-ph/0406216].

21. F. Buisseret, C. Semay, Phys. Rev. E 71, 026705 (2005) [hep-ph/0409033].

22. A. M. Badalian, B. L. G. Bakker, Phys. Rev. D 66, 034025 (2002) [hep-ph/0202246]. 
23. F. Buisseret, C. Semay, Phys. Rev. D 71, 034019 (2005) [hep-ph/0412361].

24. C. Semay, D. Baye, M. Hesse, B. Silvestre-Brac, Phys. Rev. E 64, 016703 (2001).

25. Yu. A. Simonov, Phys. Lett. B 515, 137 (2001) [hep$\mathrm{ph} / 0105141]$.

26. A. Di Giacomo, H. Panagopoulos, Phys. Lett. B 285, 133 (1992).

27. A. Di Giacomo, Yu. A. Simonov, Phys. Lett. B 595, 368 (2004) [hep-ph/0404044].

28. A. B. Kaidalov, Yu. A. Simonov, Phys. Atom. Nucl. 63, 1428 (2000) [Yad. Fiz. 63, 1428 (2000)] [hep-ph/9911291].

29. Yu. A. Simonov, hep-ph/0501182.

30. W. H. Blask et al., Z. Phys. A 337, 327 (1990).

31. F. Brau, C. Semay, Phys. Rev. D 58, 034015 (1998).

32. W. Lucha, F. F. Schöberl, Phys. Rev. Lett. 64, 2733 (1990).

33. Particle Data Group, S. Eidelman et al., Phys. Lett. B 592, 1 (2004).

34. A. Donnachie, P. V. Landshoff, Phys. Lett. B 437, 408 (1998) [hep-ph/9806344].

35. B. Silvestre-Brac, C. Semay, I. M. Narodetskii, A. I. Veselov, Eur. Phys. J. C 32, 385 (2004) [hep$\mathrm{ph} / 0309247$.

36. F. J. Llanes-Estrada, S. R. Cotanch, Phys. Lett. B 504, 15 (2001) [hep-ph/0008337].

37. M. Foster, C. Michael, Phys. Rev. D 59, 094509 (1999) [hep-lat/9811010]. 